\title{
Outcome of posterior lumbar interbody fusion in low-grade spondylolisthesis
}

\section{Vamshikrishna Chand Nimmagadda*, Hiranya Kumar Seenappa, Karthik Narayanamurthy Mittemari, Rajashree Paidipati}

Department of Orthopaedics, Vydehi Institute of Medical Sciences \& Research Center, Bengaluru, Karnataka, India

Received: 20 July 2021

Accepted: 17 August 2021

\section{*Correspondence:}

Dr. Vamshikrishna Chand Nimmagadda,

E-mail: nvkortho@hotmail.com

Copyright: () the author(s), publisher and licensee Medip Academy. This is an open-access article distributed under the terms of the Creative Commons Attribution Non-Commercial License, which permits unrestricted non-commercial use, distribution, and reproduction in any medium, provided the original work is properly cited.

\section{ABSTRACT}

Background: Spondylolisthesis is the slippage of all or part of one vertebra in relationship to a caudal vertebra, most commonly occurring in the lumbar spine. Fusion is the recommended treatment of choice. Posterior stabilization with posterior lumbar interbody fusion (PLIF) is an established procedure with good outcomes. To study functional and radiological outcomes of PLIF in grade 1 and 2 lumbar spondylolisthesis.

Methods: This is a prospective study in 30 patients who underwent PLIF and followed up for one year. Functional outcomes were measured in terms of visual analogue scale (VAS) and the Modified Oswestry Disability Index (ODI), and the fusion was assessed on plain radiographs.

Results: There was a significant improvement in VAS of low back pain $(90.71 \%, \mathrm{p}<0.001)$ and VAS of radiating $(82.52 \% ; \mathrm{p}<0.001)$. The ODI scores were significantly improved $(63.65 \%, \mathrm{p}<0.001)$ at 1 year follow up. The fusion rate was $70 \%$, and $56.66 \%$ patients reported excellent outcomes.

Conclusions: Based on functional outcomes and the fusion rates of PLIF, we conclude that PLIF is a recommended and excellent surgical option in the management of low grade lumbar spondylolisthesis.

Keywords: Spondylolisthesis, Posterior lumbar interbody fusion, Fusion, Modified Oswestry Disability index

\section{INTRODUCTION}

Spondylolisthesis refers to slippage of all or part of one vertebra in relationship to a caudal vertebra. It is most commonly seen in the lumbar spine. About $73 \%$ of all the slips are seen at L4-L5 and $18 \%$ at L3-L4. There is a female predominance with $8.1 \%$ of the patients being female and $2.7 \%$ male. ${ }^{1}$ Spondylolisthesis causes severe axial back pain, with or without radiating pain and neurogenic claudication, leading to functional disability and poor quality of life. ${ }^{2}$

The treatment of spondylolisthesis has evolved significantly in terms of procedural approaches and instrumentation. Interbody fusion provides axial stability to the spine. Anterior Lumbar Interbody Fusion (ALIF),
Posterior Lumbar interbody fusion (PLIF) or Transforaminal Lumbar interbody fusion (TLIF) and Lateral Lumbar Inter-body Fusion (XLIF) are some of the most widely accepted procedures. ${ }^{3}$ Instrumented PLIF technique decompresses the neural elements circumferentially, resulting in satisfactory clinical outcomes in lumbar spondylolisthesis. ${ }^{4}$ The aim of posterior instrumentation with PLIF is to stabilize the motion segment, decompress neural elements, restore sagittal plane translation and prevent further slip.

The objectives of our study were- To compare functional outcomes of PLIF, as assessed with a visual analogue scale (VAS) and the Oswestry disability index (ODI) before surgery and 1 year after the surgery. To assess fusion rates at 1 year after the surgery. 


\section{METHODS}

\section{Source}

The study was done in the Department of Orthopaedics, Vydehi Institute of Medical Sciences and Research Centre, Whitefield, Bangalore. Ethical committee clearance was obtained for the study and all the institutional guidelines were strictly adhered to.

\section{Patient demographics}

We enrolled 30 patients with one-level lumbar spondylolisthesis who were admitted to Vydehi Hospital for surgical treatment from September 2019 to June 2021. Inclusion criteria were- Imaging studies showing one-level spondylolisthesis, with or without lumbar disc herniation or spinal stenosis. Low back pain with or without Unilateral or bilateral lower limb pain, numbness, and or intermittent. Failed conservative management. Our standardized conservative therapy of three to six months included; use of lumbosacral corset, avoiding strenuous activities, use of Non-Steroidal Anti Inflammatory Drugs and drugs like pregabalin, nortriptyline and gabapentin for radiating pain.

Patients who had multi segment lumbar disc herniation, spinal stenosis, severe osteoporosis or other metabolic bone disease, fractured vertebra, or a congenital, isthmic or other abnormal bone structure; or infection in the intervertebral space or other areas, or who have undergone previous surgical treatment of segmental defects; or have severe systemic disease were excluded from the study.

The demographic data of the patients are summarized in Table 1.

\section{Surgical procedure}

Patients underwent a PLIF procedure using standard surgical protocol. ${ }^{5}$ First the pedicle screws were placed, followed by discectomy and disc space preparation by unilateral distractor instrumentation with bilateral curettage. Two cages were packed with cortico cancellous autografts and inserted in the prepared space. The final position of the cages was confirmed by image intensifier. Cortico-cancellous bone grafts were placed in posterolateral decorticated beds. Contoured connecting rods were fixed to the pedicle screw heads and were tightened under compression.

\section{Outcomes}

We Assessed Visual Analogue Scale (VAS) of low back pain, VAS of leg pain and Modified Oswestry disability index (ODI) at regular intervals, and compared preoperative scores with 1 year follow up scores. Radiographic fusion was assessed by Brantigan, Steffee, Fraser (BSF) classification. ${ }^{6}$

\section{Statistical analysis}

Statistical analyses were performed using Prism software (GraphPad, San Diego, CA). P value less than 0.05 is considered statistically significant. Numerical values are expressed as mean \pm standard deviation.

\section{RESULTS}

Summary of all the results are shown in Table 2.

The mean pre-operative VAS score for low back pain was $7.83 \pm 0.83$ and at one year follow up was $1.50 \pm 0.37$. There is a significant improvement $(90.71 \%, \mathrm{p}<0.0001)$ in VAS scores for back pain (Figure 1A).

Table 1: Patient demographics.

\begin{tabular}{|c|c|}
\hline \multicolumn{2}{|l|}{ Patient demographics } \\
\hline \multirow{2}{*}{ Total number of patients } & 42 years \\
\hline & 30 \\
\hline Male & $13(43 \%)$ \\
\hline Female & $17(57 \%)$ \\
\hline \multicolumn{2}{|l|}{ Level of lesion } \\
\hline L4-L5 & $18(58.3 \%)$ \\
\hline L5-S1 & $12(41.6 \%)$ \\
\hline \multicolumn{2}{|c|}{ Grade of slip (myerding classification) } \\
\hline Grade 1 & $20(66.66 \%)$ \\
\hline Grade 2 & $10(33.33 \%)$ \\
\hline
\end{tabular}

21 patients had radiating pain in our study. The mean preoperative VAS score for radiating pain was $4.75 \pm 2.59$ and at one year of follow up was $0.83 \pm 0.71$. There is a significant improvement $(82.52 \%, \mathrm{p}<0.0001)$ in VAS scores for radiating pain (Figure 1B).

The mean pre-operative ODI score was $63.5 \pm 3.27$ and at one-year follow was $22 \pm 2.3$. There is a significant improvement $(63.35 \%, \mathrm{p}<0.0001)$ in ODI scores (Figure $1 \mathrm{C})$.

Fusion was assessed following BSF Classification. Based on the classification, 21 patients had radiographical fusion (BSF 3), 7 patients had locked pseudoarthrosis (BSF2) and 2 patients had pseudoarthrosis (BSF1). Overall fusion rate is $70 \%$. (Table 2)

Overall outcome was assessed based on ODI score at 1 year follow up. There was excellent outcome in 17 patients $(56.66 \%)$ and good outcome in remaining 13 patients $(43.33 \%)$. (Figure 1D)

Pre-operative, post-operative and 1 years follow up radiographs of PLIF are shown in Figure 2. Fusion is indicated by osteointegration of interbody cage with superior and inferior end plates, (Figure 3, yellow arrow). 
Figure 1: Functional outcomes of PLIF
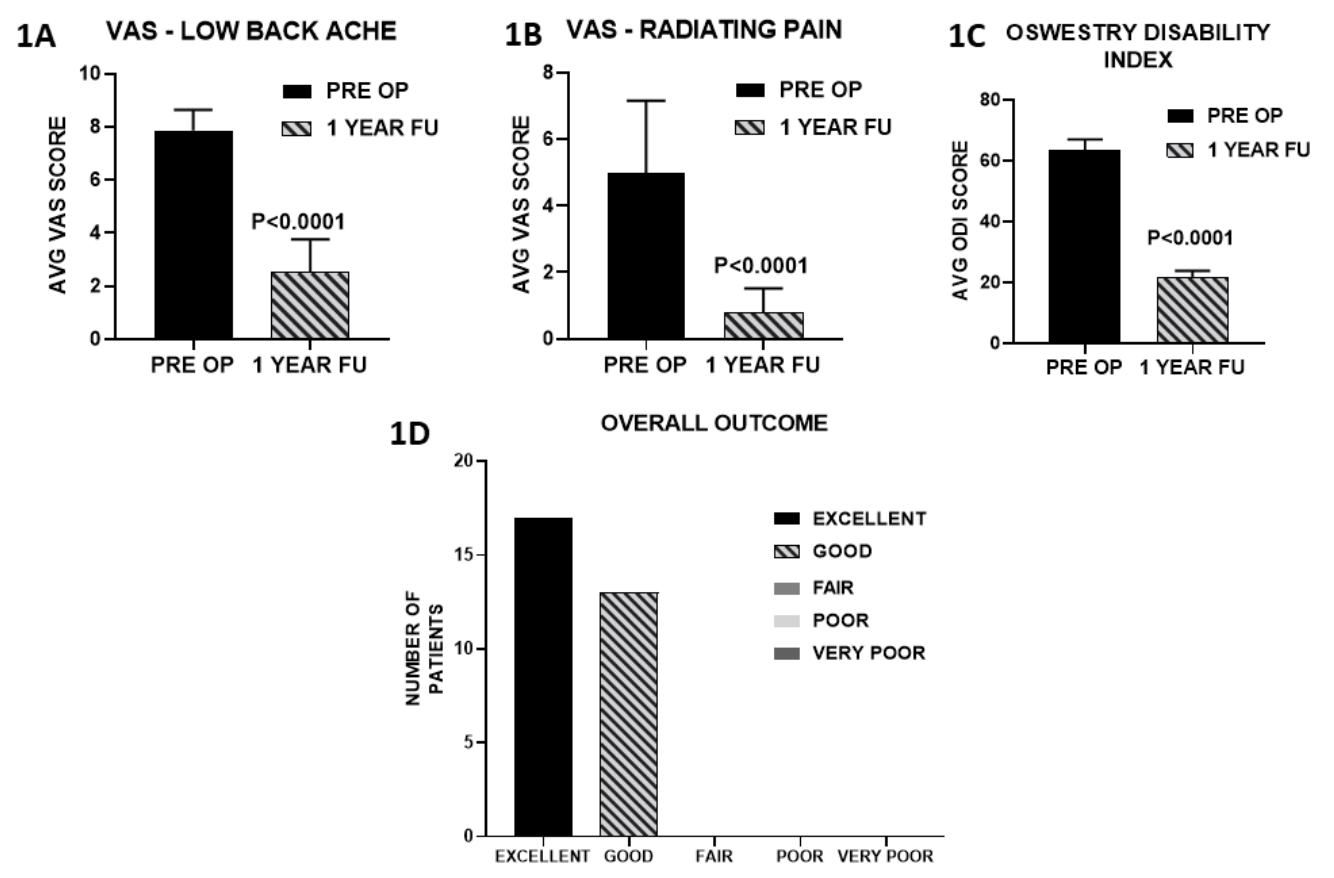

Figure 1: Functional outcomes of PLIF. (A). Quantification of VAS for low back ache. There is a statistically significant improvement in one year follow up VAS compared to pre-operative scores $(p<0.0001, N=30$, Paired $t$ test). (B). Quantification of VAS for radiating pain. There is a statistically significant improvement in one year follow up VAS compared to pre-operative scores ( $\mathbf{p}<0.0001, \mathrm{~N}=\mathbf{2 1}$, Paired $t$-test). (C). Quantification of Modified Oswestry Disability Index (ODI) scores. There is a statistically significant improvement in ODI score at 1 year follow up ( $\mathbf{p}<0.0001, \mathrm{~N}=30$, Paired t-test). (D). Overall Outcome in PLIF. Quantification of overall outcome at 1 year follow up. All the patients had either Excellent or good outcome.

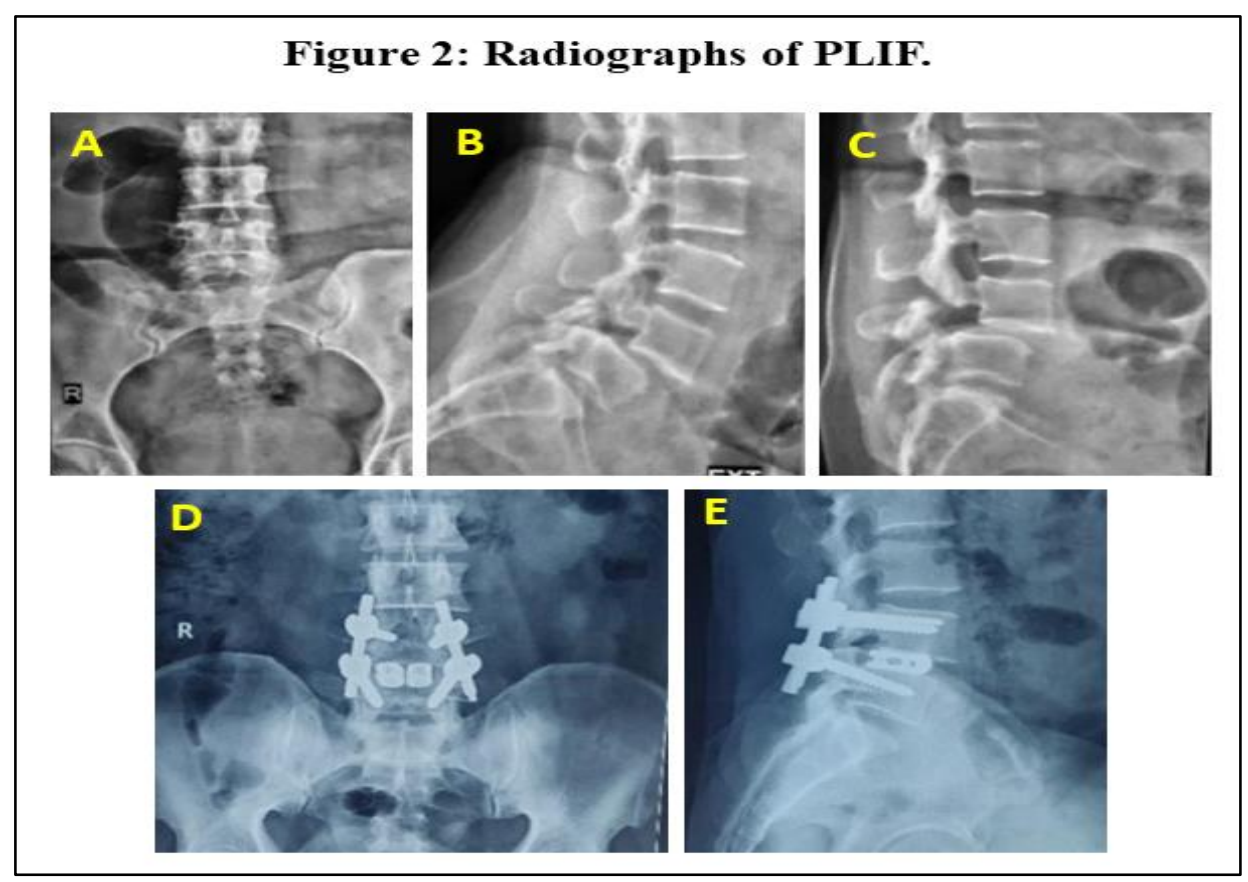

Figure 2: Radiographs of PLIF. (A, B, C) Pre-operative radiographs showing grade 2 spondylolisthesis of L4-L5 in Anteroposterior (A), lateral extension(B) and lateral flexion(C) views. (D, E) Post-operative Antero Posterior radiograph (D) showing pedicle screw fixation and interbody cage. Post-operative lateral radiograph (E) pedicle screw fixation and Interbody cage. 
There were no surgical site infections. There was dural tear during the surgery in 2 patients, which were repaired immediately. There were no neurological deficits in any of the patients after the surgery.

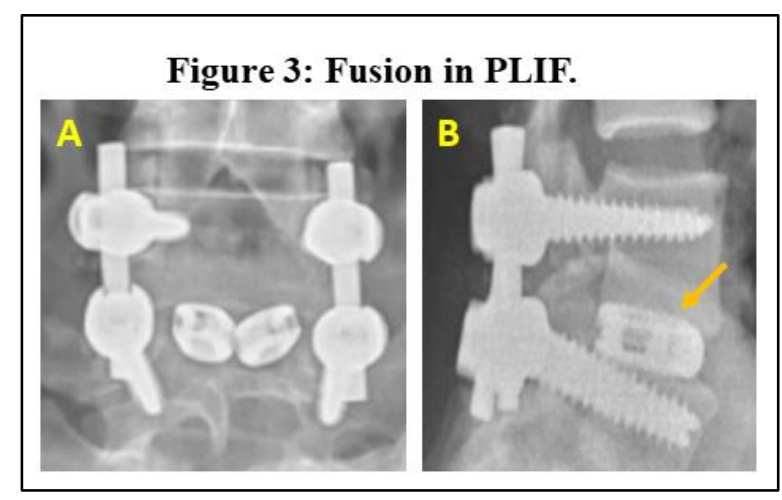

Figure 3: Fusion in PLIF. One year follow up Anteroposterior (A) and Lateral radiograph (B) radiographs. Integration of interbody cages with superior and inferior end plates indicating fusion (Yellow Arrow).

Table 2: Clinical and radiographical outcomes.

\begin{tabular}{|c|c|}
\hline \multicolumn{2}{|c|}{ Clinical and radiographical outcomes } \\
\hline \multicolumn{2}{|c|}{ Visual analog scale- low back ache $(n=30)$} \\
\hline Pre operative & $7.83 \pm 0.83$ \\
\hline 1 year follow up & $1.50 \pm 1.31$ \\
\hline Percentage improvement & $90.71 \%$ \\
\hline Significance & $\mathrm{p}<0.001$ \\
\hline \multicolumn{2}{|c|}{ Visual analog scale-radiating pain $(n=21)$} \\
\hline Pre operative & $4.75 \pm 2.59$ \\
\hline 1 year follow up & $0.83 \pm 0.71$ \\
\hline Percentage improvement & $82.52 \%$ \\
\hline Significance & $\mathrm{p}<0.001$ \\
\hline \multicolumn{2}{|c|}{ Modified oswestery disability index score $(n=30)$} \\
\hline Pre operative & $63.5 \pm 3.27$ \\
\hline 1 year follow up & $22 \pm 2.30$ \\
\hline Percentage improvement & $63.35 \%$ \\
\hline Significance & $\mathrm{p}<0.001$ \\
\hline \multicolumn{2}{|c|}{ Fusion: brantigan, steffee, fraser (BSF) grade } \\
\hline BSF 1 & $2(6.67 \%)$ \\
\hline BSF 2 & $7(30 \%)$ \\
\hline BSF 3 & $21(63.33 \%)$ \\
\hline \multicolumn{2}{|l|}{ Overall outcome } \\
\hline Excellent (ODI 0-20) & $17(56.66 \%)$ \\
\hline Good (ODI 21-40) & $13(43.33 \%)$ \\
\hline
\end{tabular}

\section{DISCUSSION}

Lumbar fusion is a successful surgical intervention for various lumbar pathologies, such as lumbar spondylolisthesis, canal stenosis, instability and discogenic pain. The primary purpose of spinal fusion surgery is to achieve a solid arthrodesis of the spinal segments with restoration of disc height, immobilization of unstable segments and restoring load bearing to anterior structures. PLIF has been widely and successfully used in the management of lumbar spondylolisthesis. ${ }^{2}$ PLIF with pedicle screw instrumentation has gained popularity around the world since introduced by Cloward in 1940s and it has been considered as the standard surgical technique for the treatment of lumbar degenerative diseases. $^{2}$

The mean age of the patients being treated for spondylolisthesis, according to the studies varied from 39.5 years to 57.4 years, with a wide range of 21 years to 70 years. $^{7}$ Our study included 30 patients with mean age being 42.83 years, with youngest patient being 32 years and oldest being 73 years. Spondylolisthesis, especially degenerative has shown more prevalence in females as was stated in a meta-analysis study conducted by Wang et al, similarly in our study we had a female predominance, 17 of the 30 subjects were females. ${ }^{8}$

Lumbar spondylolisthesis usually involves L4-L5 and L5S1 levels. L4-15 level in degenerative spondylolisthesis and L5-S1 level in isthmic spondylolisthesis., ${ }^{9,10} \mathrm{We}$ included all the patients with spondylolisthesis from age 18 to 75 years. Our study had 18 patients $(58.3 \%)$ with lesion at L4-L5 level and 12 patients at L5-S1 level $(41.6 \%)$.

The primary goal of PLIF is to achieve solid fusion. With the application of spinal instrumentation, pedicle screws provide immediate spinal stability with three columns stabilization and improve the fusion rates. Apart from interbody fusion, PLIF also provides anterior support and $360^{\circ}$ fusion. Okuda et al, conducted a study on surgical outcomes of posterior lumbar interbody fusion in elderly patients, and concluded that there was no non-union or collapsed union or delayed union. The fusion results were satisfactory. ${ }^{4}$ In another study by Meisel et al, concluded that at 6 month follow up there was confirmed evidence of fusion in PLIF, and no untoward results or outcomes noted radiologically. ${ }^{11}$ Our study demonstrates an excellent fusion rate of $70 \%$ at one year follow up.

Various studies on spondylolisthesis and have reported that the VAS scores for back pain reduced by $63 \%$ and for radiating pain reduced by $55 \%$ in patients who underwent surgical management. ${ }^{2,4,6,7,12}$ In our study, after a follow up period of 1 year, VAS score for back pain improved by $90.71 \%$ and that of radiating pain improved by $82.52 \%$.

ODI scoring system was another tool which we used in our study to measure the functional outcomes. The average ODI score at I year follow up in our study was 22 , with a $63.35 \%$ improvement. The results are consistent with studies conducted by Lan et al and Ender et al. ${ }^{2,12}$

PLIF has been reported to be associated with many complications including nerve root injury, dural tear and epidural adhesion. ${ }^{2}$ We too have encountered dural tear in 
2 patients. However, there were no incidences of any nerve root injuries or post-operative neurological deficits.

We acknowledge that we did have a few limitations in the study. One being sample size, though we did not have a large sample size, the results were statistically significant. The duration of our study was only one year, a longer duration may show higher fusion rates.

\section{CONCLUSION}

Based on our study and available literature, we conclude that posterior lumbar interbody fusion with posterior instrumentation is an excellent choice with good functional results in adults with low grade spondylolisthesis.

\section{Funding: No funding sources}

Conflict of interest: None declared

Ethical approval: The study was approved by the institutional ethics committee

\section{REFERENCES}

1. Guigui P, Ferrero E. Surgical treatment of degenerative spondylolisthesis. Orthop Traumatol Surg Res. 2017;103(1S):S11-20.

2. Lan T, Hu SY, Zhang YT, Zheng YC, Zhang R et al. Comparison Between Posterior Lumbar Interbody Fusion and Transforaminal Lumbar Interbody Fusion for the Treatment of Lumbar Degenerative Diseases: A Systematic Review and Meta-Analysis. World Neurosurg. 2018;112:86-93.

3. Nimmagadda V, Seenappa HK, Mittemari K. Outcomes of instrumented posterolateral fusion in patients with low grade lumbar spondylolisthesis. International Journal of Orthopaedics Sciences. 2020;6:139-42.

4. Shinya O, Takenori O, Akira M, Takamitsu H, Tomio Y, Motoki I. Surgical Outcomes of Posterior Lumbar Interbody Fusion in Elderly Patients, The Journal of Bone \& Joint Surgery. 2006;88(12):2714-20.
5. Williams KD. Spondylolisthesis. In: Frederick M Azar, James H Beaty, Terry Canale S. editors. Campbell's Operative Orthopaedics. 14th ed. Elsevier. 2021;1825-6.

6. Aygün H, Cakar A, Hüseyinoğlu N, Hüseyinoğlu U, Celik R. Clinical and radiological comparison of posterolateral fusion and posterior interbody fusion 95 techniques for multilevel lumbar spinal stabilization in manual workers. Asian Spine J. 2014;8(5):571-80.

7. Liu X, Wang Y, Qiu G, Weng X, Yu B. A systematic review with meta-analysis of posterior interbody fusion versus posterolateral fusion in lumbar spondylolisthesis. Eur Spine J. 2014;23:43-56.

8. Wang YXJ, Káplár Z, Deng M, Leung JCS. Lumbar degenerative spondylolisthesis epidemiology: A systematic review with a focus on gender-specific and age-specific prevalence. J Orthop Translat. 2016;11:39-52.

9. Collis JS. Total disc replacement: a modified posterior lumbar interbody fusion. Report of 750 cases. Clin Orthop Relat Res. 1985;193:64-7.

10. Wiltse LL, Winter RB. Terminology and measurement of spondylolisthesis. J Bone Joint Surg. Am. 1983;65:768- 72.

11. Meisel HJ, Schnöring M, Hohaus C, Minkus Y, Beier A, Ganey T, Mansmann U. Posterior lumbar interbody fusion using rhBMP-2. Eur Spine J. 2008;17(12):1735-44.

12. Endler P, Ekman P, Möller H, Gerdhem P. Outcomes of Posterolateral Fusion with and without Instrumentation and of Interbody Fusion for Isthmic Spondylolisthesis: A Prospective Study. J Bone Joint Surg. Am. 2017;99(9):743-52.

Cite this article as: Nimmagadda VC, Seenappa HK, Mittemari KN, Paidipati R. Outcome of posterior lumbar interbody fusion in low-grade spondylolisthesis. Int J Res Orthop 2021;7:991-5. 